Bull. Austral. Math. Soc.

VOL. 45 (1992) [415-422]

\title{
SHARP ERROR BOUNDS FOR NEWTON-LIKE METHODS UNDER WEAK SMOOTHNESS ASSUMPTIONS
}

IOANNIS K. ARGYROS

We provide sufficient convergence conditions as well as sharp error bounds for Newton-like iterations which generalise a wide class of known methods for solving nonlinear equations in Banach space.

\section{INTRODUCTION}

Let $F$ be a nonlinear operator defined on a convex subset $E_{3}$ of a Banach space $E_{1}$ with values in a Banach space $E_{2}$. A lot of methods for solving the equation

$$
F(x)=0
$$

can be written in the Newton-like form

$$
x_{n+1}=x_{n}-A\left(x_{n}\right)^{-1} F\left(x_{n}\right), \quad n \geqslant 0
$$

where for each $n \geqslant 0, A\left(x_{n}\right)^{-1}$ is a bounded linear operator from $E_{2}$ into $E_{1}$ (that is, $A\left(x_{n}\right)^{-1} \in L\left(E_{2}, E_{1}\right)$ ). Obviously the linear operator $A\left(x_{n}\right)$ must be a consistent approximation to the Fréchet-detivative $F^{\prime}$ of $F$. The best known method of type (2) are Newton's methods, where $A\left(x_{n}\right)=F^{\prime}\left(x_{n}\right)$, and the secant method, where $A\left(x_{n}\right)=\delta F\left(x_{n}, x_{n-1}\right), n \geqslant 0, \delta F$ being a consistent approximation of the Fréchetderivative of $F$. Other authors (see, for example $[1,2,3,6,7,8,9]$ and the references therein) in order to find an approximate solution $x^{*}$ of equation (1) have imposed various conditions such as

$$
\begin{gathered}
\left\|A\left(x_{0}\right)^{-1}\left(F^{\prime}(x+t(y-x))-A(x)\right)\right\| \leqslant w\left[\left(\left\|x-x_{0}\right\|+t\|y-x\|\right)^{p}\right], \\
\left\|A\left(x_{0}\right)^{-1}\left(A(x)-A\left(x_{0}\right)\right)\right\| \leqslant w_{0}\left(\left\|x-x_{0}\right\|^{p}\right)
\end{gathered}
$$

for all $x, y \in E_{3}$ and some $p, t \in[0,1]$, where $x_{0} \in E_{3}$. Here $w, w_{0}$ denote nondecreasing continuous functions from $\mid \mathbb{R}^{+}$into $\mid \mathbb{R}^{+}$with $w(0)=w_{0}(0)=0$. Denote by $N$ the class of all such functions. However these conditions do not provide sharp error estimates for Newton-like methods when $0<p<1$ (see for example $[1,2,3,6$, $7,8,9]$. In the elegant paper by Galperin and Waksman [4] sharp error bounds were found for Newton's method using the notion of a $w$-regularly continuous operator. Here we use a generalised notion of the above definition and provide sharp error bounds for Newton-like methods. Our results can be compared favourably with results already in the literature for various choices of the linear operator $A(x)$.

Received 9 May 1991

Copyright Clearance Centre, Inc. Serial-fee code: 0004-9729/92 \$A2.00+0.00. 


\section{Convergence Results}

Given an operator $G: E_{3} \subset E_{1} \rightarrow E_{2}$, and a linear operator $A(x): E_{3} \rightarrow E_{2}$, we say that $G$ is $w, A$-continuous at a point $x \in E_{3}$ if the function $w$ belongs to the class

$$
M\left(G, x, E_{3}\right):=\left\{w \in N \mid \forall y \in E_{3}\left\|A\left(x_{0}\right)^{-1}(G(y)-A(x))\right\| \leqslant w(\|x-y\|)\right\},
$$

and that $G$ is $w, A$-continuous on $E_{3}$ if $w$ belongs to

$$
M\left(G, E_{3}\right):=\left\{w \in N \mid \forall x, y \in E_{3}\left\|A\left(x_{0}\right)^{-1}(G(x)-A(y))\right\| \leqslant w(\|x-y\|)\right\} .
$$

All functions of $M\left(G, x, E_{3}\right)$ are called local continuity moduli of $G($ at $x)$, whereas those of $M\left(G, E_{3}\right)$ are called (global) continuity moduli of $G$ (on $\left.E_{3}\right)[3,6]$.

Let $N^{*}$ denote the subclass of $N$ consisting of all $w \in N$ that are concave. Denote

$$
H(x, y)=\min \left\{\left\|A\left(x_{0}\right)^{-1} G(x)\right\|,\left\|A\left(x_{0}\right)^{-1} A(y)\right\|\right\}, x, y \in E_{3} .
$$

Given $w \in N^{*}$, we say that $G$ is $w, A$-regularly continuous on $E_{3}$, if

$$
\begin{gathered}
w^{-1}\left(H(x, x+t(y-x))+\left\|A\left(x_{0}\right)^{-1}(G(x+t(y-x))-A(x))\right\|\right) \\
-w^{-1}(B(x, x+t(y-x))) \leqslant\left\|x_{0}-x\right\|+t\|y-x\|
\end{gathered}
$$

for all $x, y \in E_{3}$ and $t \in[0,1]$.

Here $w^{-1}(s)$ stands for the least root of the equation $w(t)=s$. Clearly, $w^{-1}$ is an increasing convex function defined on $[0, w(\infty))$. Because of $w^{-1}$ convexity, the above inequality implies $w \in M\left(G, E_{3}\right)$. As in [4] we can show that the converse is not always true. For $x_{0}, x, y \in E_{3}$, assume $A\left(x_{0}\right)$ is invertible and define the numbers $\alpha$, $r, \bar{a}, \bar{a}^{\prime}, \bar{b}, c, q$ by

$$
\begin{gathered}
\left\|A\left(x_{0}\right)^{-1} F\left(x_{0}\right)\right\| \leqslant \alpha, r=\|x-y\|, \bar{a}=w^{-1}\left(\left\|A\left(x_{0}\right)^{-1} A(x)\right\|\right) \\
\bar{a}^{\prime}=w^{-1}\left(\left\|A\left(x_{0}\right)^{-1} F^{\prime}(x)\right\|\right), \bar{b}=w^{-1}\left(\left\|A\left(x_{0}\right)^{-1} F^{\prime}(y)\right\|\right)-r \\
c=\left\|x-x_{0}\right\|, q=\bar{a}^{\prime}-\bar{b}
\end{gathered}
$$

the functions $q(s, t), R^{+}, B, C, D$ by

$$
\begin{aligned}
q(s, t) & =\min \{t, s-t\}, R^{+}=\max \{R, 0\}, \\
B\left(a, a^{\prime} b, c, r\right) & =\int_{0}^{r}\left[w\left(\min \left\{a,\left(a^{\prime}-q(s, t)\right)^{+}\right\}+c+t\right)-w\left(\min \left\{a,\left(a^{\prime}-q(s, t)\right)^{+}\right\}\right)\right] d t, \\
C(r) & =B\left(a(r), a^{\prime}(r), b(r), r, r\right),
\end{aligned}
$$


with (for each fixed $r \geqslant 0$ )

$$
\begin{gathered}
a=a(r)=w^{-1}\left(1-w_{0}(r)\right), b=b(r)=w^{-1}\left(1-w_{0}(r)-w(r)\right)-r \\
a^{\prime}=a^{\prime}(r)=w^{-1}\left(1-w_{0}(r)-w(r)\right)
\end{gathered}
$$

and

$$
D(r)=\alpha+\frac{C(r)}{1-w_{0}(r)}
$$

Finally, define the iteration $\left\{t_{n}\right\}, n \geqslant 0$, by $t_{0}=0, t_{1}=\alpha$ and

$$
t_{n+2}=t_{n+1}+\frac{B\left(a\left(t_{n+1}-t_{n}\right), a^{\prime}\left(t_{n+1}-t_{n}\right), b\left(t_{n+1}-t_{n}\right), t_{n}, t_{n+1}-t_{n}\right)}{1-w_{0}\left(t_{n}\right)}, n \geqslant 0 .
$$

We can now state and prove the main result:

Theorem. Let $F: E_{3} \subset E_{1} \rightarrow E_{2}$ and $w \in N^{*}$ Assume:

(i) There exist $x_{0} \in E_{3}$ and a positive number $\alpha$ such that $A\left(x_{0}\right)$ is invertible and $\left\|A\left(x_{0}\right)^{-1} F\left(x_{0}\right)\right\| \leqslant \alpha$.

(ii) There exists a minimum positive number $r * \in\left(0, w_{0}^{-1}(1)\right)$ such that

$$
D(r) \leqslant r \text { and } 1-w_{0}(r)-w(r) \geqslant 0 \text { for all } f \in\left(0, r^{*}\right]
$$

(iii) $U=U\left(x_{0}, r^{*}\right)=\left\{x \in E_{1} \mid\left\|x-x_{0}\right\| \leqslant r^{*}\right\} \subset E_{3}$.

(iv) Given $A(x) \in L\left(U, E_{2}\right)$ satisfying (4) for $p=1$ for all $x \in U$, let $F$ be Fréchet differentiable on $U$ and $F^{\prime}$ be $w-A$ regularly continuous on $U$.

Then,

(1) the function $B$ does not increase in each of its first three arguments and increases in the other two;

(2) the iteration $\left\{t_{n}\right\}, n \geqslant 0$ is increasing and bounded above by $r^{*}$ with $t^{*}=\lim _{n \rightarrow \infty} t_{n} \leqslant r^{*}$;

(3) the operator $A(x)$ is invertible on $U$;

(4) the Newton-like iterations (2) are well defined, remain in $U\left(x_{0}, t^{*}\right)$ and converge to a solution $x^{*}$ of equation (1);

(5) $x^{*}$ is the unique solution of equation (1) in $U\left(x_{0}, r^{*}\right)$;

(6) the following estimates are true:

$$
\begin{gathered}
\left\|x_{n+1}-x_{n}\right\| \leqslant t_{n+1}-t_{n} \\
\left\|x_{n}-x^{*}\right\| \leqslant t^{*}-t_{n} \text { for all } n \geqslant 0
\end{gathered}
$$


(9)

$$
\leqslant \frac{B\left(a\left(\left\|x_{n}-x_{n-1}\right\|\right), a^{\prime}\left(\left\|x-n-x_{n-1}\right\|\right), b\left(\left\|x_{n}-x_{n-1}\right\|\right),\left\|x_{n-1}-x_{0}\right\|,\left\|x_{n}-x_{n-1}\right\|\right)}{1-w_{0}\left(\left\|x^{*}-x_{0}\right\|\right)}
$$

for all $n \geqslant 1$

(10)

$$
\begin{gathered}
\left\|x_{n+1}-x_{n}\right\| \leqslant\left\|x_{n}-x^{*}\right\| \\
+\frac{B\left(a\left(\left\|x^{*}-x_{n}\right\|\right), a^{\prime}\left(\left\|x^{*}-x_{n}\right\|\right), b\left(\left\|x^{*}-x_{n}\right\|\right),\left\|x_{n}-x_{0}\right\|,\left\|x^{*}-x_{n}\right\|\right)}{1-w_{0}\left(\left\|x_{n}-x_{0}\right\|\right)}
\end{gathered}
$$

for all $n \geqslant 0$.

(7) the convergence condition (6) and the estimates (7)-(9) are sharp.

Proof: (1) The proof of this part is similar to the corresponding one in $[4$, Lemma 2.1] and so is omitted.

(2) The first two members of the iteration $\left\{t_{n}\right\}, n \geqslant 0$ are such that $t_{0}<t_{1} \leqslant r^{*}$. Therefore the denominator of the fraction appearing in the definition of the sequence is positive. That is, $t_{1} \leqslant t_{2}$ (since the numerator is obviously nonnegative). Let us assume that $t_{k} \leqslant t_{k+1}, k=0,1,2, \ldots, n$. Then by the definition of the sequence, $\left\{t_{n}\right\}, n \geqslant 0, t_{k+1} \leqslant t_{k+2}$. That is, $t_{n+1} \leqslant t_{n+2}$ for $n=k+1$. So far we have shown that the scalar sequence $\left\{t_{n}\right\}$ is increasing for all $n \geqslant 0$. We will show that $t_{n} \leqslant r^{*}$ for all $n \geqslant 0$. For $n=0,1$ this is true by hypothesis. For $n=2, t_{2} \leqslant r^{*}$, since $t_{2} \leqslant D\left(r^{*}\right) \leqslant r^{*}$. Let us assume that $t_{k} \leqslant r^{*}, k=0,1,2, \ldots, n$; then

$$
C\left(t_{1}-t_{0}\right)+C\left(t_{2}-t_{1}\right)+\ldots+C\left(t_{k+1}-t_{k}\right) \leqslant C\left(t_{k+1}-t_{0}\right) \leqslant C\left(t_{t+1}\right) \leqslant C\left(r^{*}\right),
$$

since the function $w$ is increasing and $\left(t_{1}-t_{0}\right)+\left(t_{2}-t_{1}\right)+\ldots+\left(t_{k+1}-t_{k}\right)=t_{k+1}-t_{0}$. Hence $t_{k+1} \leqslant C\left(r^{*}\right) \leqslant r^{*}$, which completes the induction. Therefore the sequence $\left\{t_{n}\right\}$, $n \geqslant 0$ is increasing and bounded above by $r^{*}$ and as such it converges to some $t^{*}$ such that $0<t^{*} \leqslant r^{*}$.

(3) Let us observe that the linear operator $A(u)$ is invertible for all $u \in U\left(x_{0}, w_{0}^{-1}(1)\right)$. Indeed we obtain

$$
\left\|A\left(x_{0}\right)^{-1}\left(A(u)-A\left(x_{0}\right)\right)\right\| \leqslant w_{0}\left(\left\|u-x_{0}\right\|\right)<1,
$$

so that according to Banach's lemma $A(u)$ is invertible and

$$
\left\|A(u)^{-1} A\left(x_{0}\right)\right\| \leqslant\left(1-w_{0}\left(\left\|u-x_{0}\right\|\right)\right)^{-1} \text {. }
$$

Note also that since $\left\|A\left(x_{0}\right)^{-1} A(u)\right\| \cdot\left\|A(u)^{-1} A\left(x_{0}\right)\right\| \geqslant 1$, then $\left\|A\left(x_{0}\right)^{-1} A(u)\right\| \geqslant$ $1-w_{0}\left(\left\|u-x_{0}\right\|\right)$. 
(4)-(6) It now follows that if (2) is well defined for $n=1,2,3, \ldots, k$ and if (7) holds for $n \leqslant k$ then

$$
\left\|x_{0}-x_{n}\right\| \leqslant t_{n}-t_{0} \leqslant t^{*}-t_{0} \text { for } n \leqslant k .
$$

This shows that (7) is satisfied for $u=x_{i}$ with $i \leqslant k$. Thus (2) is well defined for $n=k+1$ too. Also from $\left\|x_{0}-x_{k}\right\| \leqslant t_{k}-t_{0} \leqslant t^{*}$ we obtain $x_{k} \in U\left(x_{0}, t^{*}\right)$.

We now observe that (7) is true for $n=0$. Assume that it is true for $k=$ $0,1,2, \ldots, n$. Then by (2)

$$
\begin{aligned}
& \left\|x_{k+2}-x_{k+1}\right\|=\left\|A\left(x_{k+1}\right)^{-1} F\left(x_{k+1}\right)\right\| \\
= & \left\|A\left(x_{k+1}\right)^{-1}\left(F\left(x_{k+1}\right)-F\left(x_{k}\right)-A\left(x_{k}\right)\left(x_{k+1}-x_{k}\right)\right)\right\| \\
= & \left\|A\left(x_{k+1}\right)^{-1} A\left(x_{0}\right)\right\| \cdot\left\|A\left(x_{0}\right)^{-1}\left(F\left(x_{k+1}\right)-F\left(x_{k}\right)-A\left(x_{k}\right)\left(x_{k+1}-x_{k}\right)\right)\right\| \\
= & \left\|A\left(x_{k+1}\right)^{-1} A\left(x_{0}\right)\right\| \cdot\left\|A\left(x_{0}\right)^{-1}\left[\int_{0}^{1} F^{\prime}\left(x_{k}+F\left(x_{k+1}-x_{k}\right)\right)-A\left(x_{k}\right)\right]\left(x_{k+1}-x_{k}\right) d t\right\| .
\end{aligned}
$$

We now apply (11) for $u=x_{k+1},(5)$ for $x=x_{k}, y=x_{k+1}, G=F^{\prime}$ to obtain (13)

$$
\begin{aligned}
& \left\|x_{K+2}-x_{K+1}\right\| \\
& \leqslant \frac{1}{1-w_{0}\left(t_{k}\right)} \int_{0}^{1}\left[w\left(w^{-1}\left(H\left(x_{k}, x_{k}+t\left(x_{k+1}-x_{k}\right)\right)+\left\|x_{k}-x_{0}\right\|+\left\|x_{k+1}-x_{k}\right\| t\right)\right)\right. \\
& \left.\quad-w\left(w^{-1}\left(H\left(x_{k}, x_{k}+t\left(x_{k+1}-x_{k}\right)\right)\right)\right)\right]\left\|x_{k+1}-x_{k}\right\| d t \\
& \leqslant \frac{B\left(a\left(t_{k+1}-t_{k}\right), a^{\prime}\left(t_{k+1}-t_{k}\right), b\left(t_{k+1}-t_{k}\right), t_{k}, t_{k+1}-t_{k}\right)}{1-w_{0}\left(t_{k}\right)}=t_{k+2}-t_{k+1}
\end{aligned}
$$

This shows (7) for $n=k+1$. Hence, $\left\{x_{n}\right\}, n \geqslant 0$ is a Cauchy sequence in a Banach space and as such it converges to a point $x^{*} \in U$. By (12) and (13) we observe that the numerator of $(13)$ is an upper bound for $\left\|A\left(x_{0}\right)^{-1} F\left(x_{k+1}\right)\right\|$ which tends to 0 as $k \rightarrow \infty$. Hence, by continuity, $F\left(x^{*}\right)=0$. The estimate (8) now follows easily from (7).

To show uniqueness, let us assume that there exist two solutions $x^{*}$ and $y^{*}$ in $U\left(x_{0}, r^{*}\right)$ and consider the estimate $F\left(x^{*}\right)-F\left(y^{*}\right)=L^{*}\left(x^{*}-y^{*}\right)$ with $L^{*}=$ $\int_{0}^{1} F^{\prime}\left(y^{*}+t\left(x^{*}-y^{*}\right)\right) d t$.

Then as before (see (11)) we can show $\left\|I-A\left(x_{0}\right)^{-1} L^{*}\right\|<1$. That is, $L^{*}$ is invertible, which shows $x^{*}=y^{*}$. 
Set $L=\int_{0}^{1} F^{\prime}\left(x^{*}+t\left(x_{n}-x^{*}\right)\right) d t$ and use (11), and the estimates

$$
\begin{aligned}
\left\|x_{n}-x^{*}\right\| & \leqslant\left\|\left(A\left(x_{0}\right)^{-1} L\right)^{-1}\right\| \cdot\left\|A\left(x_{0}\right)^{-1} F\left(x_{n}\right)\right\|, \\
\left\|x_{n+1}-x_{n}\right\| & =\left(x^{*}-x_{n}\right)+\left(A\left(x_{0}\right)^{-1} A\left(x_{n}\right)\right)^{-1} \\
& {\left[A\left(x_{0}\right)^{-1}\left(F\left(x^{*}\right)-F\left(x_{n}\right)-A\left(x_{n}\right)^{-1}\left(x^{*}-x_{n}\right)\right)\right] }
\end{aligned}
$$

to obtain (9) and (10) respectively.

(7) This follows exactly as in part (5) Theorem 2.1 in [4], which completes the proof of the theorem.

It can easily be seen that if $w(t)=\gamma t, w_{0}(t)=\beta t$ for some $\beta, \gamma>0$ and the sequence $\left\|x^{*}-x_{n}\right\|$ is monotone then (9) and (10) can provide an upper and a lower bound on $\left\|x^{*}-x_{n}\right\|$ respectively expressed in terms of the rest of the norms. Moreover define the numbers $r_{1}, r_{2}, r_{3}, \Delta$ and the intervals $I_{1}, I_{2}, I$ by

$$
r_{1}=\frac{1}{\beta+\gamma}, \quad r_{2}=\frac{1+\alpha \beta-\sqrt{\Delta}}{3 \gamma+2 \beta}, \quad r_{3}=\frac{1+\alpha \beta+\sqrt{\Delta}}{3 \gamma+2 \beta},
$$

with $\Delta=(1+\alpha \beta)^{2}-2 \alpha(3 \gamma+2 \beta), I_{1}=\left(0, r_{1}\right), I_{2}=\left[r_{2}, r_{3}\right]$, and $I=I_{1} \cap I_{2}$.

Assume:

$$
\Delta>0 \text { and } I \neq \emptyset \text { and set } I_{3}=\left[r_{2}, \min \left(r_{1}, r_{3}\right)\right] .
$$

It can easily be seen then that condition (6) is satisfied for all $r \in I_{3}$.

Similar conditions can be obtained when $w_{0}(t)=\beta t^{p}, w(t)=\gamma t^{p}$ for $p \in[0,1)$. In the latter case the results in $[3,5,7,8,9]$ cannot apply (since $p=1$ there). Moreover it can easily be seen that our results compare favourably with the ones in $[1,2,3,6]$ in this case.

Finally, consider the equation

$$
F(x)+Q(x)=0
$$

where $F$ is as before and $Q$ is a nonlinear operator defined on $E_{3}$ with values on $E_{2}$ such that

$$
\left\|A\left(x_{0}\right)^{-1}(Q(x)-Q(y))\right\| \leqslant w_{1}(\|x-y\|) \text { for all } x, y \in E_{3}
$$

for some nondecreasing real function $w_{1}$ defined on $R^{+}$with $w_{1}(0)=0$. Note that the differentiability of $Q$ is not assumed. Define the function $D_{1}(r)$ by

$$
D_{1}(r)=\alpha+\frac{C_{1}(r)}{1-w_{0}(r)}, \quad C_{1}(r)=C(r)+w_{1}(r)
$$


and the iteration $\left\{s_{n}\right\}, n \geqslant 0$, by $s_{0}=0, s_{1}=\alpha$ and

$$
\begin{aligned}
s_{n+2}= & s_{n+1} \\
+ & \frac{B\left(a\left(s_{n+1}-s_{n}\right), a^{\prime}\left(s_{n+1}-s_{n}\right), b\left(s_{n+1}-s_{n}\right), s_{n}, s_{n+1}-s_{n}\right)+w_{1}\left(s_{n+1}-s_{n}\right)}{1-w_{0}\left(s_{n}\right)}, \\
& \quad n \geqslant 0
\end{aligned}
$$

Then with the rest of the notation as before we can immediately state and prove a theorem for approximating a solution $x^{*}$ of equation (14) similar to the one above. Just replace $D(r)$ by $D_{1}(r)$ and $\left\{t_{n}\right\}$ by $\left\{s_{n}\right\}, n \geqslant 0$, in the above theorem and take into account hypothesis (15).

Note that the iteration (2) will become

$$
z_{n+1}=z_{n}-A\left(z_{n}\right)^{-1}\left(F\left(z_{n}\right)+Q\left(z_{n}\right)\right), z_{0} \in E_{3}, n \geqslant 0 .
$$

The new theorem will cover the case when the operator appearing in equation (1) is not Fréchet-differentiable but it can be decomposed into one that is and one that is not (see also [2] and the references therein).

\section{REFERENCES}

[1] I.K. Argyros, 'Newton-like methods under mild differentiability conditions with error analysis', Bull. Austral. Math. Soc. 37 (1988), 131-147.

[2] X. Chen and T. Yamamoto, 'Convergence domains of certain iterative methods for solving nonlinear equations', Numer. Funct. Anal. Optim. 10 (1989), 37-48.

[3] J.E. Dennis, 'Toward a unified convergence theory for Newton-like methods', in Nonlinear functional analysis and applications, Editor L.B. Rall, pp. 425-472 (Academic Press, New York, 1971).

[4] A. Galperin and Z. Waksman, 'Newton's method under a weak smoothness assumption', J. Comput. Appl. Math. (to appear).

[5] L.V. Kantorovich and G.P. Akilov, Functional analysis in normed spaces (Pergamon Press, Oxford, 1964).

[6] G.J. Miel, 'Unified error analysis for Newton-type methods', Numer. Math. 33 (1979), 391-396.

[7] F.A. Potra, 'On the convergence of a class of Newton-like methods', in Iterative solution of nonlinear systems of equations: Lecture notes in Math. 953, Editors R. Ansorge, T. Meis and W. Torning, pp. 125-137 (Springer-Verlag, Berlin, Heidelberg, New York, 1982).

[8] W.C. Rheinboldt, 'A unified convergence theory for a class of iterative process', SIAM J. Numer. Anal. 5 (1968), 42-63.

[9] T. Yamamoto, 'A convergence theorem for Newton-like methods in Banach spaces', $N u$ mer. Math. 51 (1987), 545-557. 
Department of Mathematics

Cameron University

Lawton OK 73505

United States of America 